\title{
Nonequilibrium phase transition to a time-dependent probability density for a model of charge-density waves
}

\author{
Luis L. Bonilla \\ Departamento de Física Teórica, Universidad de Sevilla, Apartado Correos 1.065, Sector Sur, E-41080 Sevilla, Spain
}

(Received 18 June 1986; revised manuscript received 10 September 1986)

\begin{abstract}
A nonequilibrium phase transition to a stable time-periodic one-particle probability density is found in a modified Fukuyama-Lee model of charge-density waves. The classical single-phase model of Grüner, Zawadowski, and Chaikin is derived in the zero-temperature limit as the equation for the order parameter of this transition. Two-time correlations are given as functionals of the order parameter. These results raise the question of the existence of stable nonequilibrium probability densities in other models. Stable time-dependent densities are shown to exist for replicas of general dynamical systems having a stable time-dependent attractor in the zero-noise limit. This is so if the replicas are coupled via a mean-field interaction and the thermodynamic limit is taken.
\end{abstract}

\section{INTRODUCTION}

Some time ago, Desai and Zwanzig ${ }^{1}$ studied critical dynamics of the mean-field version of the $\phi^{4}$ theory. They derived a time-dependent nonlinear Fokker-Planck equation for the one-particle probability density in the thermodynamic limit. Dawson ${ }^{2}$ proved this equation to be exact. The phase transition appeared as a pitchfork bifurcation for the equilibrium probability density when the temperature (which is the square of the strength of the thermal noise) was varied. This result could never have been obtained in a standard linear Fokker-Planck equation, because the $H$ theorem implies the uniqueness and stability of the equilibrium.

For a modification of the Fukuyama-Lee ${ }^{3}$ model of charge-density waves (CDW's), another nonlinear Fokker-Planck equation holds in the thermodynamic limit. This time, a different kind of phase transition is found in the zero-temperature limit: As the external field surpasses a critical value, the one-phase probability density changes from an equilibrium distribution to a stable time-periodic (nonequilibrium) distribution. Furthermore, the order parameter of this nonequilibrium phase transition obeys the single-phase equation of Grüner, Zawadowski, and Chaikin. ${ }^{4}$ From the order parameter, one- and two-time correlations can be obtained. These correlations cannot be obtained from the original deterministic model of Ref. 4.

The results for the simplified Fukuyama-Lee model raise the question of the existence of nonequilibrium probability densities and nonequilibrium phase transitions in general models. We give a partial answer: Let $d x /$ $d t=f(x)$ be a dynamical system having a time-dependent attractor $x=A(t)$. Add a vanishingly small white-noise forcing term to it. Consider $N$ replicas of the resultant stochastic system coupled via a mean-field interaction in the limit $N \rightarrow \infty$, the one-system probability density tends (as $t \rightarrow \infty)$ to a time-dependent functional of $A(t)$.

\section{THE SIMPLIFIED FUKUYAMA-LEE MODEL}

A discretized mean-field version of the Fukuyama-Lee model $^{5}$ of CDW's consists of the following equations

$$
\begin{aligned}
& (j=1, \ldots, N) \text { : } \\
& d \phi_{j} / d t=E-h_{j} \sin \left(\phi_{j}-\beta_{j}\right) \\
& -J\left(\phi_{j}-N^{-1} \sum_{1}^{N} \phi_{k}\right)+F^{1 / 2} w_{j}(t) \text {. }
\end{aligned}
$$

Here $\phi_{j}$ is the slowly varying phase of the CDW at the site $j$. $J$ is the stiffness of the CDW and $E$ the applied electric field. $h_{j}$ and $\beta_{j}$ represent the random impurity potential and pinning angle, respectively. The Gaussian white noise $w_{j}(t) \quad\left[\left\langle w_{j}\right\rangle=0,\left\langle w_{i}(t) w_{j}\left(t^{\prime}\right)\right\rangle=\delta_{i j} \delta\left(t-t^{\prime}\right)\right]$ contains the effect of the temperature. Equation (1) is due to Fisher. ${ }^{5}$ We want to consider the case $h_{j}=h>0$ and $\beta_{j}=0$ in (1). The random external fields introduced by Fisher ${ }^{5}$ thus disappear. As we will show elsewhere, ${ }^{6}$ the resultant simplified model is still useful to analyze (1) in the strongpinning regime, where $J \ll \bar{h}$.

Suppose that the initial $N$-phase probability density corresponding to (1) (with $h_{j}=h>0$ and $\beta_{j}=0$ ) is a product of $N$ one-phase probability densities $\mu\left(\phi_{j}\right), j=1, \ldots, N$. We have then proved that, asymptotically as $N \rightarrow \infty$, the one-phase probability density $p(t, \phi)$ obeys the following nonlinear equations: ${ }^{7}$

$$
\begin{aligned}
& \partial_{t} p(t, \phi)=\frac{1}{2} F \partial_{\phi}^{2} p(t, \phi) \\
& -\partial_{\phi}\{[E-h \sin \phi+J(\bar{\phi}(t)-\phi)] p(t, \phi)\}, \\
& \bar{\phi}(t)=\int_{0}^{2 \pi} \phi p(t, \phi) d \phi, \\
& p(0, \phi)=\mu(\phi) \text {, } \\
& \int_{0}^{2 \pi} p(t, \phi) d \phi=1 \text {. }
\end{aligned}
$$

Equation (2) has the form of a Fokker-Planck equation, except that the coefficient $\bar{\phi}(t)$ is a functional of the density. Once (2) is solved for an arbitrary function $\bar{\phi}(t)$, the consistency condition (3) acts as a bifurcation equation, as we show below. The solution of (2) and (3) has the symmetry

$$
p(t, \phi ; \bar{\phi}(t))=p(t, \phi+2 \pi ; \bar{\phi}(t)+2 \pi) .
$$

We use this symmetry as a boundary condition and solve 


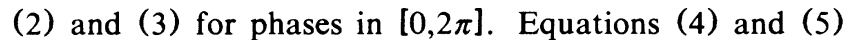
are initial and normalization conditions, respectively.

Asymptotically for $F$ near zero, the stationary solutions of (2) and (3) have the form ${ }^{8}$

$$
\begin{aligned}
& p_{s}(\phi ; \bar{\phi})=e^{-2 V(\phi ; \bar{\phi}) / F}\left(\int_{0}^{2 \pi} e^{-2 V(\psi ; \bar{\phi}) / F} d \psi\right)^{-1}, \\
& V(\phi ; \bar{\phi})=J(\phi ; \bar{\phi})^{2}-E \phi-h \cos \phi \\
& \bar{\phi}=\int_{0}^{2 \pi} \phi p_{s}(\phi ; \bar{\phi}) d \phi .
\end{aligned}
$$

The integrals in (6) and (8) may be asymptotically evaluated by Laplace's method for $F$ near zero. We have to find the minimum of $V(\phi ; \bar{\phi})$ for $\phi$ in $[0,2 \pi]$. For a fixed $\bar{\phi}$, $\partial_{\phi} V(\phi ; \bar{\phi})=0$ has zero, one, two, or three different solutions in $[0,2 \pi]$, according to the relative values of the parameters $\bar{\phi}+E / J$ and $h / J$. Of these solutions, only one will, in general, minimize $V(\phi ; \bar{\phi})$. Let us call it $\phi_{0}$. Then $\bar{\phi} \sim \phi_{0}$ according to (8). Inserting this consistency condition in $\partial_{\phi} V(\phi ; \bar{\phi})=0$, we find that

$$
E=h \sin \phi_{0} \text {. }
$$

For $E<h$, this equation has only one solution in $[0, \pi / 2]$, which minimizes $V(\phi ; \bar{\phi})$. For $E>h,(9)$ has no solution. Where then does an initial density evolve as $t \rightarrow \infty$ ?

To solve $(2)-(5)$ in the low-temperature limit $(F \rightarrow 0)$, we use the Wentzel-Kramers-Brillouin (WKB) method, thereby inserting in (2) the following function:

$$
\begin{aligned}
p(t, \phi)= & \exp [-\Psi(t, \phi) / F] \\
& \times\left[Z_{0}(t, \phi)+F Z_{1}(t, \phi)+O\left(F^{2}\right)\right] .
\end{aligned}
$$

We find an eikonal equation for $\Psi$ and linear transport equations for the $Z$ 's. The eikonal equation is

$\partial_{t} \Psi+\frac{1}{2}\left(\partial_{\phi} \Psi\right)^{2}+\{E-h \sin (\phi-\beta)+J[\bar{\phi}(t)-\phi]\} \partial_{\phi} \Psi=0$

$\bar{\phi}(t)$ in (11) is given by (3) and (10). The solution of the initial-value problem for $\Psi$ is attained by the method of characteristics. It is

$$
\Psi(t, \phi ; h, \beta)=\Omega(\Sigma(\phi, t))+\frac{1}{2}\left[\Omega^{\prime}(\Sigma(t, \phi))\right]^{2} \int_{0}^{t} d s \exp \left(2 J s+2 h \int_{0}^{s} \cos [\Phi(\Sigma(\phi, t), r)-\beta] d r\right)
$$

Here $\quad \Omega(\phi)=\Psi(0, \phi) \sim-F \ln \mu(\phi), \quad \Omega^{\prime}(\phi) \equiv d \Omega(\phi) / d \phi$, and $\Phi(s, t)$ is the solution of the characteristic equations

$$
\begin{aligned}
d \Phi(s, t) / d t= & E-h \sin (\Phi-\beta)+J[\bar{\phi}(t)-\Phi]+\Omega^{\prime}(s) \\
& \times \exp \left(J t+h \int_{0}^{t} \cos [\Phi(s, r)-\beta] d r\right), \\
\Phi(s, 0)=s . &
\end{aligned}
$$

Once $\Phi(s, t)$ is known, $\Sigma(\phi, t)$ is its inverse function (if it exists):

$$
\Phi(\Sigma(\phi, t), t)=\phi, \Sigma(\Phi(s, t), t)=s .
$$

To evaluate $\bar{\phi}(t)$ we use Laplace's method in (8). Asymptotically in the limit $F \rightarrow 0, \bar{\phi}(t)$ is equal to the function $\phi=\phi_{0}(t)$ which minimizes $\Psi(t, \phi)$. Thus $\bar{\phi}(t)$ satisfies

$$
\partial_{\phi} \Psi(t, \bar{\phi}(t))=0 \text {. }
$$

Assuming that $\Sigma(\phi, t)$ exists, and considering (12), (16) is satisfied if $\Omega^{\prime}[\Sigma(\bar{\phi}(t), t)]=0$. Suppose that $\sigma$ is an extremum of the initial-value function $\Omega(s)$; think of an initial probability density peaked at $\phi=\sigma$, for example. Then $\Sigma(\bar{\phi}(t), t)=\sigma$, which gives $\Phi(\sigma, t)=\bar{\phi}(t)$. By insertion in (13) and (14), we find

$$
d \bar{\phi}(t) / d t=E-h \sin \bar{\phi}(t), \bar{\phi}(0)=\sigma .
$$

Equation (17) is just the single-phase model of Grüner, Zawadowski, and Chaikin. ${ }^{4}$ For $E \leq h$, there are two stationary solutions of $(17), \phi_{0}$ and $\pi-\phi_{0}$, where $\phi_{0}$ is the value (9). $\phi_{0}$ is an asymptotically stable solution of (17) while $\pi-\phi_{0}$ is unstable. At $E=h, \phi_{0}=\pi / 2$, and the two stationary solutions coalesce. For $E>h$, there are no stationary solutions of (17): For any $\sigma, \bar{\phi}(t)$ is periodic, with period $T=2 \pi\left(E^{2}-h^{2}\right)^{-1 / 2}$. Which $p(t, \phi)$ do the solutions of (17) correspond to for different values of $E / h$ ?

For $E \leq h, \bar{\phi}(t)$ tends to $\phi_{0}$, and $p(t, \phi)$ tends to the sta- tionary density $p_{s}(\phi ; \bar{\phi})$ of (6). For $E>h, \bar{\phi}(t)$ is periodic and we can calculate an approximation to the density $p(t, \phi)$ as follows. Suppose $E$ is larger than, but not too close to, $h$. Consider the following time-periodic Gaussian density,

$$
\begin{gathered}
p(t, \phi) \sim \exp \left\{-|\phi-\bar{\phi}(t)|^{2} /[F W(t)]\right. \\
\left.-O\left(|\phi-\bar{\phi}(t)|^{3}\right)\right\} .
\end{gathered}
$$

This corresponds to having the exponent

$$
-|\phi-\bar{\phi}(t)|^{2} /[F W(t)]+O\left(|\phi-\bar{\phi}(t)|^{3}\right)
$$

in the WKB solution (10). Let us insert this functional in the eikonal equation (11), and then expand

$$
\sin \phi=\sin \bar{\phi}(t)+\cos \bar{\phi}(t)[\phi-\bar{\phi}(t)]+O\left(|\phi-\bar{\phi}(t)|^{2}\right) .
$$

By ignoring $O\left(|\phi-\bar{\phi}(t)|^{3}\right)$ terms, we find the equation for $W(t)$,

$$
d W(t) / d t+J W(t)=1+\cos \bar{\phi}(t) .
$$

This equation has the following $T$-periodic solution:

$$
\begin{aligned}
& W(t)=\gamma(t)[1-\gamma(T)]^{-1} \int_{t-T}^{t} d s / \gamma(s), \\
& \gamma(t)=\exp \left(-2 J t-2 h \int_{0}^{t} \cos \bar{\phi}(s) d s\right) .
\end{aligned}
$$

Equations (18) and (20) form a nonuniform approximation of $p(t, \phi)$ which is asymptotically valid for $\phi$ near $\bar{\phi}(t)$ and $F \rightarrow 0 . W(t)$ is approximately equal to the correlation $\left\langle\phi^{2}(t)\right\rangle$. Near $E=h, W(t)$ diverges. We need to keep higher-order terms in (18), typically up to third-order terms. $^{9}$

Two-time correlations $\left\langle\phi(t) \phi\left(t^{\prime}\right)\right\rangle$ are calculated by means of

$\left\langle\phi_{i}(t) \phi_{j}\left(t^{\prime}\right)\right\rangle=\delta_{i j} \int_{0}^{2 \pi} \int_{0}^{2 \pi} \phi \psi p^{c}\left(t-t^{\prime} ; \phi, \psi\right) d \phi d \psi$. 
The conditional density $p^{c}(t ; \phi, \psi)$ satisfies Eqs. (2), (3), and (5), with the initial condition (22) below instead of (4):

$$
p^{c}(0 ; \phi, \psi)=\delta(\phi-\psi) .
$$

Laplace's method applied to (21) yields

$$
\left\langle\phi_{i}(t) \phi_{j}\left(t^{\prime}\right)\right\rangle \sim \delta_{i j} \int_{0}^{2 \pi} \psi \bar{\phi}(t ; \psi) d \psi .
$$

$\bar{\phi}(t ; \psi)$ obeys Eq. (17) with initial condition $\bar{\phi}(t ; \psi)=\psi$.

\section{A GENERAL RESULT FOR MEAN-FIELD MODELS IS IN THE WEAK-NOISE LIMIT}

Let us prove here the claim made in the Introduction concerning dynamical systems $d x / d t=f(x)$, with an attractor $A(t)$. We now add a white noise term to $f(x)$, and analyze the corresponding equation (1) with $f\left(x_{j}\right)$, substituting $E-h_{j} \sin \left(\phi_{j}-\beta_{j}\right)$. The analysis of Sec. II goes through with trivial modifications. It yields the equation $d \bar{x}(t) / d t=f(\bar{x}(t)), \bar{x}(0)=\sigma$, for the mean value of $x$. As $A(t)$ is the asymptotically stable solution of this equation, $\bar{x}(t) \rightarrow A(t)$ as $t \rightarrow \infty$. The probability density will tend to a time-dependent functional of $A(t)$ which is of the form (18) for $x$ close to $A(t)$. It seems reasonable to ask that the local correlation $W(t)$ have the same features as $A(t)$. Further work in this direction [for an aperiodic $A(t)$, for example, a strange attractor] seems interesting.

\section{DISCUSSION}

We have analyzed a simplified Fukuyama-Lee model of CDW's in the low-temperature limit. This model retains the mean-field interaction among the phase of the CDW at different sites, but it ignores the external noise due to the impurities. In the thermodynamic limit, we obtain a nonlinear equation for a single-phase probability density. ${ }^{7}$ An asymptotic analysis valid for $F \rightarrow 0$ (vanishing temperature), yields the classical equation (17), for the mean phase of the CDW. ${ }^{4}$ Thus the single-phase model of Ref. 4 can be derived from a model still containing collective effects due to the interaction among phases at different sites. Had we included the effect of the noises, we would have obtained Fisher's equation (4.4), ${ }^{5}$ with a different mean- ing of the variables: The phase $\phi$ is now a collective coordinate which minimizes the WKB exponent $\Psi$, not any single phase $\phi_{j}$ as in Ref. 5. This difference turns out to be crucial for the analysis. ${ }^{6}$ Assuming (as we do here) that the phases of the deterministic pinning potential are coherent $\left(\beta_{j}=0, h_{j}=h\right)$, our analysis shows the following.

(a) To the extent that the classical model of Ref. 4 is reasonable ${ }^{10}$ the depinning of the CDW is a nonequilibrium phase transition; the probability density changes from a stationary equilibrium distribution to a time-periodic one.

(b) Our statistical analysis yields expressions for the mean and different correlations of the phase of the CDW. All of them are periodic with the same period as the mean.

(c) The critical exponents for our model are, of course, those calculated in Ref. 4. They are incompatible with experiments. To get better results we must study Eq. (1) including the noises $h_{j}$ and $\beta_{j}{ }^{6}$

Furthermore, we have shown that nonequilibrium timedependent probability densities are conspicuous in meanfield models: In the weak-noise limit, we can build timedependent densities out of any dynamical system having a time-dependent attractor. The main requirements for this are the mean-field interaction among replicas of the dynamical system, the molecular chaos initial condition for the $N$-replica probability density, and the thermodynamic limit. If extrapolation from this result is legitimate, nonequilibrium phase transitions present in the weak-noise limit should also be present for finite temperature. We have checked this statement in a model of synchronization of oscillators, where standard Hopf bifurcation techniques work for any noise strength. ${ }^{11}$

Note added in proof. When $V(\phi ; \bar{\phi})$ has no minimum for a fixed $\bar{\phi}$, there is a solution of the form (6), which has a single maximum at $\phi=2 \pi$. This stationary solution, which is overlooked here, is crucial to explaining why there are no sliding CDW's at high temperatures.

\section{ACKNOWLEDGMENTS}

The author is indebted to Dr. W. Wolff for introducing him to CDW models and for collaboration in related works, to Professor J. B. Keller for helpful comments, and to Professor M. Morillo for valuable discussions and for bringing Refs. 1 and 2 to his attention.
${ }^{1}$ R. Desai and R. Zwanzig, J. Stat. Phys. 19, 1 (1978).

${ }^{2}$ D. A. Dawson, J. Stat. Phys. 31, 29 (1983).

${ }^{3}$ H. Fukuyama and P. A. Lee, Phys. Rev. B 17, 535 (1977).

${ }^{4}$ G. Grüner, A. Zawadowski, and P. M. Chaikin, Phys. Rev. Lett. Lett. 46. 511 (1981).

${ }^{5}$ D. S. Fisher, Phys. Rev. B 31, 1396 (1985).

${ }^{6}$ L. L. Bonilla (unpublished).

${ }^{7}$ L. L. Bonilla, J. Stat. Phys. (to be published).

${ }^{8}$ The difference between (6) and the true $2 \pi$-periodic density is exponentially small as $F \rightarrow 0$, namely, $O(\exp [-4 \pi J$ $\times(\pi+\bar{\phi}) / F])$ physically, the probability current between each period [which is needed to guarantee the periodicity of $\left.p_{s}(\phi ; \bar{\phi})\right]$ is exponentially small, and it can safely be ignored, as we did in (6).

${ }^{9} \mathrm{This}$ is due to the symmetry of the saddle-node bifurcation, in which two stationary states coalesce and disappear. See M. Mangel, SIAM J. Appl. Math. 36, 544 (1979), where the corresponding (linear) Fokker-Planck equation is analyzed. In our case we must supplement Mangel's analysis with the consistency condition (3).

${ }^{10}$ G. Grüner and A. Zettl, Phys. Rep. 119, 117 (1985).

${ }^{11}$ L. L. Bonilla, J. M. Casado, and M. Morillo (unpublished). 